\title{
Spatio-temporal cluster detection of chickenpox in Valencia, Spain in the period 2008-2012
}

\author{
Adina Iftimi, ${ }^{1}$ Francisco Martínez-Ruiz, ${ }^{2}$ Ana Míguez Santiyán, ${ }^{3}$ Francisco Montes ${ }^{1}$ \\ ${ }^{1}$ Department of Statistics and Operations Research, University of Valencia; \\ ${ }^{2}$ Statistics Office, City Council of Valencia; ${ }^{3}$ Public Health and Preventive Medicine Service, \\ University Hosptial Dr. Peset, Valencia, Spain
}

\begin{abstract}
Chickenpox is a highly contagious airborne disease caused by Varicella zoster, which affects nearly all non-immune children worldwide with an annual incidence estimated at 80-90 million cases. To analyze the spatiotemporal pattern of the chickenpox incidence in the city of Valencia, Spain two complementary statistical approaches were used. First, we evaluated the existence of clusters and spatio-temporal interaction; secondly, we used this information to find the locations of the spatio-temporal clusters via the space-time permutation model. The first method used detects any aggregation in our data but does not provide the spatial and temporal information. The second method gives the locations, areas and time-frame for the spatio-temporal clusters. An overall decreasing time trend, a pronounced 12-monthly periodicity and two complementary periods were observed. Several areas with high incidence, surrounding the center of the city were identified. The existence of aggregation in time and space was observed, and a number of spatio-temporal clusters were located.
\end{abstract}

Correspondence: Adina Iftimi, Department of Statistics and Operations Research, Faculty of Mathematics, University of Valencia, C/Doctor Moliner 50, 46100 Burjassot, Spain. Tel: +34.963 .543 .989 .

E-mail: adina.iftimi@uv.es

Key words: Varicella zoster virus; Chickenpox; Airborne disease epidemiology; Spatio-temporal inhomogeneous $K$-function.

Acknowledgements: the data used in this work have been provided by the Red de Vigilancia Epidemiológica de la Comunidad Valenciana. We thank and appreciate the help, efforts and advices of all the professionals who are part of this network.

Funding: Adina Iftimi's research is funded by the Ministry of Education, Culture and Sports grant FPU12/04531. The work of Francisco Martínez-Ruiz and Francisco Montes was partially supported by grants from the Ministry of Economy and Competitiveness MTM2013-45381 and MTM2013-43917.

Received for publication: 26 February 2015.

Accepted for publication: 15 April 2015.

(C) Copyright A. Iftimi et al., 2015

Licensee PAGEPress, Italy

Geospatial Health 2015; 10:341

doi:10.4081/gh.2015.341

This article is distributed under the terms of the Creative Commons Attribution Noncommercial License (by-nc 3.0) which permits any noncommercial use, distribution, and reproduction in any medium, provided the original author(s) and source are credited.

\section{Introduction}

The Varicella zoster virus causes two different clinical forms: i) chickenpox (varicella), a primary infection characterized by a generalised rash; and ii) herpes zoster, a commonly painful reactivation of latent $V$. zoster virus infection, affecting limited areas of the body. Chickenpox is a highly contagious airborne disease that spreads easily through coughing or sneezing of infected individuals or through direct contact with secretions from rashes. Varicella affects nearly all nonimmune children worldwide with an annual incidence estimated at 80 90 million cases. Over $90 \%$ of the cases occur during the first 15 years of life. In 2005, varicella vaccination was introduced in Spain through a national program for 10-14 years old non-immune persons (Peña Rey et al., 2009).

Varicella is characterized by fever and vesicular-papular skin eruptions. It causes relatively rare complications, mainly associated with immunocompetent children. However, when varicella affects teenagers or adults the symptomatology may be less benign, commonly with severe, clinical, respiratory, neurological and/or cardiac complications in immunocompromised patients. In temperate countries, most people become infected before adulthood and 10\% of young adults remain susceptible. The incubation period ranges between 2 and 3 weeks, usually 13 to 17 days. Susceptible people should be considered infectious 10 to 21 days after infection is over. Infection gives extended immunity. There are two types of cases, suspicious/probable and confirmed cases. The suspicious cases correspond to the disease clinically consistent. The confirmed case has to meet the clinical case definition criterions or has to be confirmed by a medical laboratory.

Vaccination, the most effective measure of prevention, was introduced in the Valencian Community for all children at age 11, provided that they have not suffered the disease or been vaccinated against chickenpox previously. In 2012, the incidence rate of chickenpox in this community was 291.41 cases per 100,000 inhabitants.

The transmission of infection can be prevented by isolating the infected patient. The exclusion of the children from school has to be for at least 5 days after the rash appears or until vesicles become dry. Strict isolation in hospitals is essential to prevent severe infection of immunocompromised patients.

Several varicella studies concerning the epidemiology of $V$. zoster virus infection have been done in various parts of the world (Brisson et al., 2001). Some regard the management of varicella vaccination programs (Peña Rey et al., 2009; Ozaki, 2013), while others have focused on the impact of varicella vaccination (Al-Tawfiq et al., 2013; Gil-Prieto et al., 2014). Space-time methodology has been increasingly used over the last years (e.g. Diggle et al., 2005; Gabriel and Diggle, 2009; Diggle, 2014).

The aim of this paper is to detect spatio-temporal interaction and locate spatio-temporal clusters. In order to do this, we used two com- 
plementary statistical approaches: one described by Gabriel and Diggle (2009) and applied by Gabriel et al. (2010), and a second one proposed by Kulldorff (2010). We analysed the spatial and temporal component of our data separately, using the methodology from Diggle (2014), after which we assessed for spatio-temporal clustering. Following this, we used the space-time permutation model (Kulldorff, 2010) to find spacetime clusters based on information provided by the previous analysis. Thus, the first method helped us to detect the existence of aggregation in our data, while the second gave us the spatial location and the time periods when the clusters were observed.

\section{Materials and Methods}

We used a sample of weekly chickenpox cases that were collected over a 5 -year period in the city of Valencia, the third most populated city in Spain and located on the Mediterranean coast $\left(39^{\circ} 28^{\prime} 0^{\prime \prime} \mathrm{N}\right.$, $\left.0^{\circ} 22^{\prime} 30^{\prime \prime} \mathrm{W}\right)$. The study region consists of a central area of Valencia where the average age of the population is high, 43.4 years, and a peripheral area around the center of the city where the average age is lower, 40.9 years. Figure 1 shows all the districts of Valencia, the center of which consists of districts 1,2 and 3 .

The cases were defined as infections with sudden onset, moderate fever, minimum general symptoms and maculopapular skin rash for a few hours transforming into vesicles over the next 3 or 4 days leaving granular crusts (Servicio de Vigilancia y Control Epidemiológico, 2007). Laboratory-confirmed patients (positive for varicella) can either be identified as varicella or as herpes zoster cases. All suspicious/probable or confirmed cases of the latter were excluded from the study.

\section{Temporal analysis}

The pattern of weekly counts showed a marked annual periodicity. Therefore, an exploratory analysis of the evolution over time was carried out by fitting a harmonic regression, which models the logarithm of the intensity by means of the following equation:

$\log y(t)=\sum_{k=1}^{m}\left(\sum_{i=1}^{n}\left(\alpha_{k} \cos i k \pi t+\beta_{k} \sin i k \pi t\right)\right)+\gamma t$, for $t=1, \ldots, T$

where $y(t)$ is the temporal intensity, $\alpha_{\mathrm{k}}$ and $\beta_{\mathrm{k}}$ are the parameters of the regression, $\mathrm{g}$ is the overall trend, and $\mathrm{T}$ is the number of temporal units considered.

\section{Spatial analysis}

We investigated large-scale variation in risk using a non-parametric estimation method proposed by Diggle et al. (2005). They developed a non-parametric method to confront spatial segregation in a multivariate point process. As suggested by the authors we used their methodology for case-control data. Cases and controls are considered as partial realizations of spatially inhomogeneous Poisson point processes with intensities $\lambda_{\text {cases }}(s)$ and $\lambda_{\text {controls }}(s)$ and where $s=(x, y)$ indicates the spatial location. Using the equation:

$$
\mathrm{p}(\mathrm{s})=\lambda_{\text {cases }}(\mathrm{s}) /\left(\lambda_{\text {cases }}(\mathrm{s})+\lambda_{\text {controls }}(\mathrm{s})\right)
$$

eq. 2

we expressed the relative risk at the location s. We estimated the rela- tive risk from the data using non-parametric binary regression. Monte Carlo methods were used to test the null hypothesis of constant spatial

risk as $\mathrm{H}_{0}: \mathrm{p}(\mathrm{s})=\alpha$, where $\alpha$ can be estimated by, $\widehat{\alpha}=n /\left(n+n_{0}\right) v$ representing the number of cases and $\eta_{0}$ the number of controls. Hence, we used the following statistic test:

$T_{s}=2 \sum_{i=1}^{n_{0}+n}\left(\widehat{p}\left(s_{i}\right)-\widehat{\alpha}\right)^{2}$

where the $P$ value of the test is obtained by randomly relabelling cases and controls to generate a null distribution of $\mathrm{T}_{\mathrm{s}}$.

\section{Space-time analysis}

For the space-time analysis, $\mathrm{X}=\left(\left(\mathrm{s}_{\mathrm{i}}, \mathrm{t}_{\mathrm{i}}\right), \mathrm{i}=1,2, \ldots\right)$ denotes a point process, where $s_{i} \in R^{2}$ is the spatial location of the $i^{\text {th }}$ event, and $t_{i} \in$ $\mathrm{R}^{+}$is the time of occurrence. The study region is $\lambda=\mathrm{S} \times \mathrm{T}$, where $\mathrm{S}$ is a planar region and $\mathrm{T}$ a time interval, both $\mathrm{S}$ and $\mathrm{T}$ are bounded and $\mathrm{Y}(\mathrm{l})$ denotes the number of events in the region $\lambda$. The process is orderly (multiple coincident events cannot occur). The space-time intensity function is given by the equation:

$$
\lambda(s, t)=\lim _{|d s \times d t| \rightarrow 0} \frac{\mathbb{E}[Y(d s \times d t)]}{|d s \times d t|}
$$

where ds $\times$ dt defines a small region around the point $(s, t)$ and $|d s \times d t|$ its volume.

The second-order intensity function is defined as:

$$
\lambda_{2}\left((\boldsymbol{s}, t),\left(\boldsymbol{s}^{\prime}, t^{\prime}\right)\right)=\lim _{|d \boldsymbol{s} \times d t|,\left|d \boldsymbol{s}^{\prime} \times d t^{\prime}\right| \rightarrow 0} \frac{\mathbb{E}\left[Y(d \boldsymbol{s} \times d t) Y\left(d \boldsymbol{s}^{\prime} \times d t^{\prime}\right)\right]}{|d \boldsymbol{s} \times d t|\left|d \boldsymbol{s}^{\prime} \times d t^{\prime}\right|}
$$

and the pair correlation function as:

$$
g\left((s, t),\left(s^{\prime}, t^{\prime}\right)\right)=\frac{\lambda_{2}\left((s, t),\left(s^{\prime}, t^{\prime}\right)\right)}{\lambda(s, t) \lambda\left(s^{\prime}, t^{\prime}\right)}
$$

Second-order stationarity is achieved if the first-order and the second-order properties are invariant under translation. Baddeley et al. (2010) defines a weaker form of stationarity for spatial point processes called second-order intensity-reweighted stationarity. This allows for non-constant intensity, which is required to be bounded away from zero assuming the pair correlation function to be invariant under translation. A spatio-temporal point process is second-order intensity reweighted stationary and isotropic if its intensity function is bounded away from zero and its pair correlation function depends only on the spatiotemporal difference vector $(v, v)$, where $v=\left\|\left.\right|_{-}-\mathbf{s}^{\prime}\right\|$ and $v=\left|\mathbf{t}-\mathrm{t}^{\prime}\right|$.

A similarA similar concept to the spatial K-function (Ripley, 1981) can be defined for a second-order intensity reweighted stationary and isotropic, spatio-temporal point process, the space-time inhomogeneous K-function (STIK function), i.e.:

$$
K_{S T}(u, v)=2 \pi \int_{0}^{v} \int_{0}^{u} g\left(u^{\prime}, v^{\prime}\right) u^{\prime} d u^{\prime} d v^{\prime}
$$

where $g(v, v)=g(u, v)=\lambda_{2}(u, v) / \lambda(s, t) \lambda\left(s^{\prime}, t^{\prime}\right), v=\left\|s-s^{\prime}\right\|$ and $v=$ It-t'l. 
The STIK function characterizes the second-order properties of a second-order intensity reweighted stationary spatio-temporal point process and can be used to test for space-time clustering and spacetime interaction by comparing the estimation of the STIK function with estimations calculated under the suitable null hypothesis. For a Poisson process $K_{S T}(u, v)=\pi u^{2} v$. Values of $K_{S T}(u, v)>\pi u^{2} v$ indicate clustering at spatial and temporal separations less than $v$ and $v$, while values of $K_{S T}(u, v)<\pi u^{2} v$ indicate regularity. We can also use the space-time inhomogeneous K-function to measures interaction because in absence of interaction, $\frac{K_{S T}(u, v)}{K_{S}(u) K_{T}(v)}$ is constant (1 for a Poisson process).

To estimate the STIK function, Gabriel and Diggle (2009) propose an approximately unbiased estimator

$$
\hat{K}_{S T}(u, v)=\frac{1}{|S \times T|} \frac{n}{n_{v}} \sum_{i=1}^{n_{v}} \sum_{j=1 ; j>i}^{n_{v}} \frac{1}{w_{i j}} \frac{1}{\lambda\left(\boldsymbol{s}_{i}, t_{i}\right) \lambda\left(\boldsymbol{s}_{j}, t_{j}\right)} 1_{\left\{\left\|\boldsymbol{s}_{i}-\boldsymbol{s}_{j}\right\| \leq u ; t_{j}-t_{i} \leq v\right\}}
$$

where $\mathrm{S} \times \mathrm{T}$ represents the spatio-temporal region with $\mathrm{S}$ as an arbitrary polygon and $T=\left[T_{0}, T_{1}\right],\left(s_{i}, t_{i}\right)$ are ordered so that $t_{i}<t_{i+1}$. For each $n, u_{t}$ denotes the number of events for which $\mathrm{t}_{\mathrm{i}} \mathrm{T}_{1}-\mathrm{n}$ and $\mathrm{W}_{\mathrm{ij}}$ and is the proportion of the circle centered on $\mathrm{s}_{\mathrm{i}}$ and passing through $\mathrm{s}_{\mathrm{j}}$, i.e. of radius $\mathrm{u}_{\mathrm{ij}}=\left\|s_{\mathrm{i}}-\mathrm{s}_{\mathrm{j}}\right\|$, that lies inside $\mathrm{S}$.

For the estimation of the intensity $\lambda$, the assumption that first-order effects are separable, $\lambda(\mathrm{s}, \mathrm{t})=\mathrm{m}(\mathrm{s}) \mathrm{y}(\mathrm{t})$, for all $(\mathrm{s}, \mathrm{t}) \in \mathrm{S} \times \mathrm{T}$, is made. Estimations of $\mathrm{m}(\mathrm{s})$ and $\mathrm{Y}(\mathrm{t})$ depend on the attributes of each problem. For the space-time clustering, simulations of an inhomogeneous Poisson process with intensity $\lambda=\mathrm{m}(\mathrm{s}) \mathrm{y}(\mathrm{t})$ are compared with the data. For the space-time interaction hypothesis, the data is compared with simulations obtained by randomly relabelling the spatial locations si, holding the times $t_{i}$ fixed.

The spatio-temporal inhomogeneous K-function measures the distribution of pairs of cases that occur less than or equal to $h$ metres of distance and $\tau$ days apart. A complementary method is the space-time permutation model defined by Kulldorff (2010). This method gives us the tools to locate spatial and temporal areas with high incidence (clusters). It compares the number of observed cases in a cluster to the expected number of cases under the hypothesis of no space-time interaction. In other words, a geographical area is considered a cluster if the number of cases, during a specific time period, is higher than the number of cases of all remaining areas. This method cannot be used to find purely temporal and purely spatial clusters. It does not allow for population adjustment, that is, some areas with a higher population increase (or decrease) during the time-period, can lead to artificial clusters due to population shift rather than a disease outbreak. As our data is a realization of a five-year process, this problem is not present. The two methods are complementary to the extent that the first one detects aggregations and their spatial-temporal dimension, while the second one gives the geographical locations of the aggregations.

\section{Results}

In our study we considered both suspicious and confirmed chickenpox cases collected from the first week of 2008 to the last week of 2012 representing residents in Valencia with varicella. The data had been gathered by the General Direction of Public Health, Epidemiological Area, Vigilance Service and Epidemiological Control, Generalitat Valenciana. The majority of the population and cases of varicella were registered in districts 1 to 16 , the remaining districts, 17 to 19 , have been eliminated from the study because they are districts with very low population and far from the urban core. The number of cases registered in these districts represent only $2 \%$ of the total. Table 1 displays the evolution of the total population, the population under 15 years and the number of cases in the region study from 2008 to 2011. Due to low variation between the population of each year we choose as reference 2010 , with a number of 769,011 inhabitants.

A total of 10,293 cases were registered. Table 2 gives us information about the percentage of population by age group living in the central area of the city in comparison with those living in the periphery. We can see that for the first two age groups the percentage of population is higher in the periphery than in the central urban area.

Figure $2 \mathrm{~A}$ shows the cumulative number of cases over the 260 weeks. We observed a decreasing trend in the incidence over the 5-year period studied. Figure 2B displays the distribution of cases according to group of age and sex, and shows no significant difference between males and females. The age distribution presents two high peaks, which confirms that chickenpox is primarily a disease of children. Figure 3 shows the spatial distribution of varicella cases over the study region, for each year.

\section{Temporal analysis}

Using the harmonic regression given in equation 1 , the best results were obtained for $n=5$ and $m=2$ (see equation 2) indicating a pronounced 12 -month periodicity and two complementary periods, one from winter to spring and a second one in summer, as we can see in Figure 4.

$\log y(t)=\sum_{k=1}^{5}\left(\sum_{i=1}^{2}\left(\alpha_{k} \cos i k \pi t+\beta_{k} \sin i k \pi t\right)\right)+\gamma t$, for $t=1, \ldots, 260$

Table 3 shows the regression parameters, standard errors and 95\% confidence intervals. Every year, most likely due to school contact, the Table 1. Number of cases of chickenpox and temporal evolution in Valencia districts 1-16.

\begin{tabular}{lccccc} 
Year/demography & 2008 & 2009 & 2010 & 2011 & 2012 \\
Total population & 768,790 & 773,856 & 769,011 & 759,211 & 757,938 \\
Population <15 & 116,929 & 118367 & 118,471 & 118,144 & 118,487 \\
\hline Number of cases & 2545 & 2152 & 2438 & 1898 & 1260 \\
\hline
\end{tabular}

Table 2. Population in 2010 by age group.

\begin{tabular}{|c|c|c|c|c|c|c|c|c|c|c|}
\hline Area & $0-4$ & $5-14$ & $15-24$ & 25-34 & $\begin{array}{c}\text { Age } \\
35-44\end{array}$ & $45-54$ & $55-64$ & $65-74$ & $>75$ & Total \\
\hline Central area & $5571(4.6)$ & $9504(7.9)$ & $10,252(8.6)$ & $18,069(15.1)$ & $19,996(16.7)$ & $16,560(13.8)$ & $13,374(11.2)$ & $11,519(9.6)$ & $15,049(12.6)$ & 119,894 \\
\hline Periphery & $32,580(5.0)$ & $57,859(8.9)$ & $66,509(10.2)$ & $106,944(16.5)$ & $109,093(16.8)$ & $91,593(14.1)$ & 73,720 (11.4) & $56,386(8.7)$ & $54,433(8.4)$ & 649,117 \\
\hline Total Valencia & 38,151 & 67,363 & 76,761 & 125,013 & 129,089 & 108,153 & 87,094 & 67,905 & 69,482 & 769,011 \\
\hline
\end{tabular}

Values in brackets represent percentages. 
number of varicella cases starts to increase in October and reaches a maximum in spring. The fitted incidence is lowest in August most than likely due to summer holidays.

The overall incidence has a decreasing trend over the five-year period; the slope of the fitted trend has the value of -0.14 . This decreasing trend can be explained by the vaccination calendar of the Valencian Community, which was modified in February of 2006 when the varicella vaccination was introduced for 11-years-old non-immune children.

\section{Spatial clustering}

The study region consists of a central area, where the average age of the population is high and a peripheral area around the center of the city where the average age is lower. Table 2 shows the population in 2010 with the center of Valencia less populated than the rest of the city, which means that, in addition to the low average age, there are more susceptible persons living in the peripheral area. To test the hypothesis that the incidence is proportional to population density we considered a sample of controls. To select the controls we used the Population Census from January 1 of 2010 (Statistics Office of Valencia Local Council, 2010). We selected $n_{0}=4 n$ controls randomly sampled from the population at risk, where $n$ represents the total number of cases. We tested for spatial clustering for each year separately and for all 5 years jointly. Table 4 indicates that the hypothesis of constant risk was reject- ed both for every year and for the 5-year study period $(\mathrm{P}<0.05)$, confirming that varicella incidence is not proportional to the population density. Figure 5 shows local $P$ values associated with the hypothesis

Table 3. Estimation of the regression parameters.

\begin{tabular}{lcccc} 
Parameters & Estimate & Standard error & $2.5 \%$ CI & $97.5 \%$ CI \\
Intercept & 2.303 & 0.047 & 2.211 & 2.394 \\
$\alpha 1$ & -0.067 & 0.015 & -0.097 & -0.037 \\
\hline$\alpha 2$ & 0.480 & 0.017 & 0.447 & 0.513 \\
$\beta 1$ & 1.160 & 0.022 & 1.117 & 1.204 \\
\hline$\beta 2$ & -0.227 & 0.016 & -0.259 & -0.196 \\
Trend & 0.026 & 0.001 & 0.024 & 0.028 \\
\hline
\end{tabular}

Table 4. Annual significance values associated with spatial clustering.

\begin{tabular}{lcccccc} 
& All years & 2008 & 2009 & 2010 & 2011 & 2012 \\
Pvalue & 0.001 & 0.001 & 0.001 & 0.001 & 0.001 & 0.001 \\
\hline
\end{tabular}
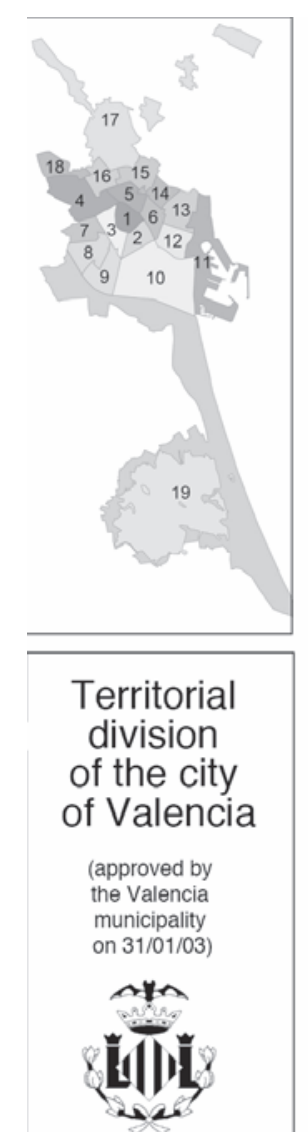

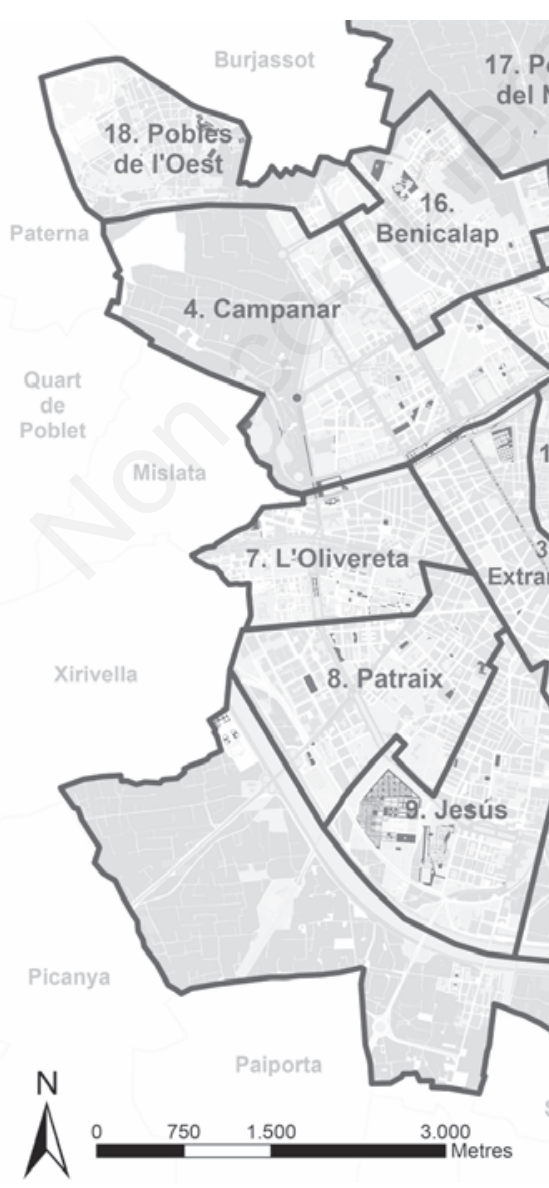

Figure 1. Valencia city map and study region; districts 1 to 16 . 
for the 5-year time period and estimated individually for each year. Darker areas correspond to value $<0.025$ and show significantly higher spatial incidence than average. We identified several areas with significant elevation in the number of cases relative to the number of controls, surrounding the center of the city. In the South of Valencia, areas with significantly high incidence correspond to neighbourhoods with a high concentration of lower-middle class population, which often do not meet with the vaccination schedules. These areas also have low density population and high number of cases. Figure 5 shows some areas east of Valencia that had high risk of varicella in 2011. These areas matches with a neighbourhood that has the same characteristics concerning the population as those in the south of the city. For the years 2008, 2009 and 2010, Figure 5 further shows several clusters corresponding to areas with a socio-economic situation less favorable and with younger populations than the downtown neighbourhoods.

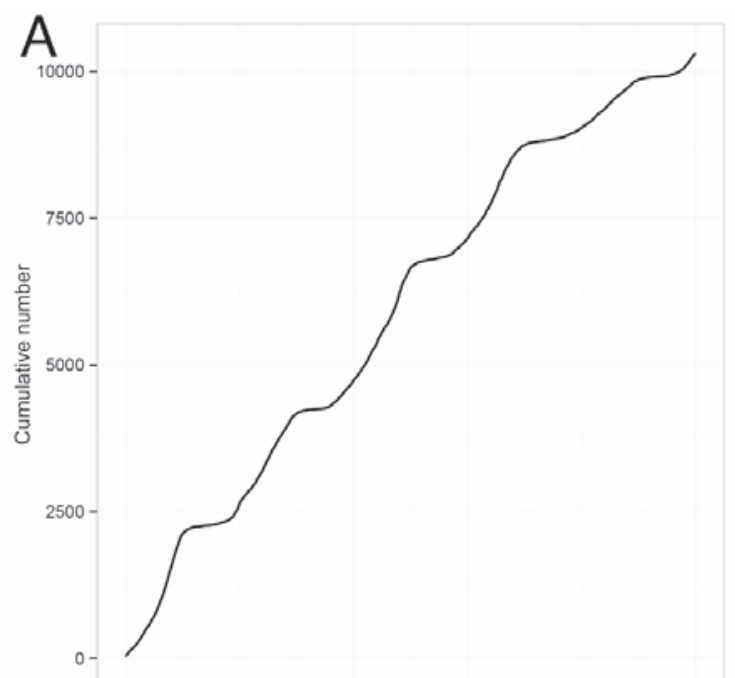

week $1 / 2008$ week ${ }^{1} / 2009$ week ${ }^{1} / 2010$ week $1 / 2011$ week ${ }^{1}$ week 2012 week 52/2012

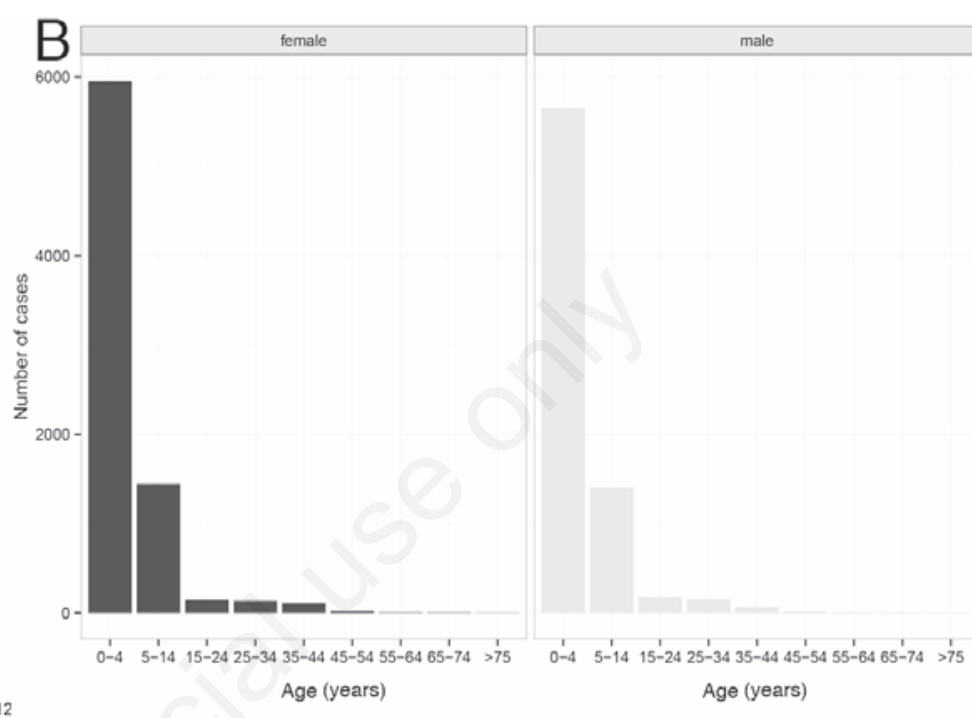

Age (years)

Figure 2. A) Cumulative, temporal distribution of the number of chickenpox cases; B) number of cases by age group and gender during the study period.
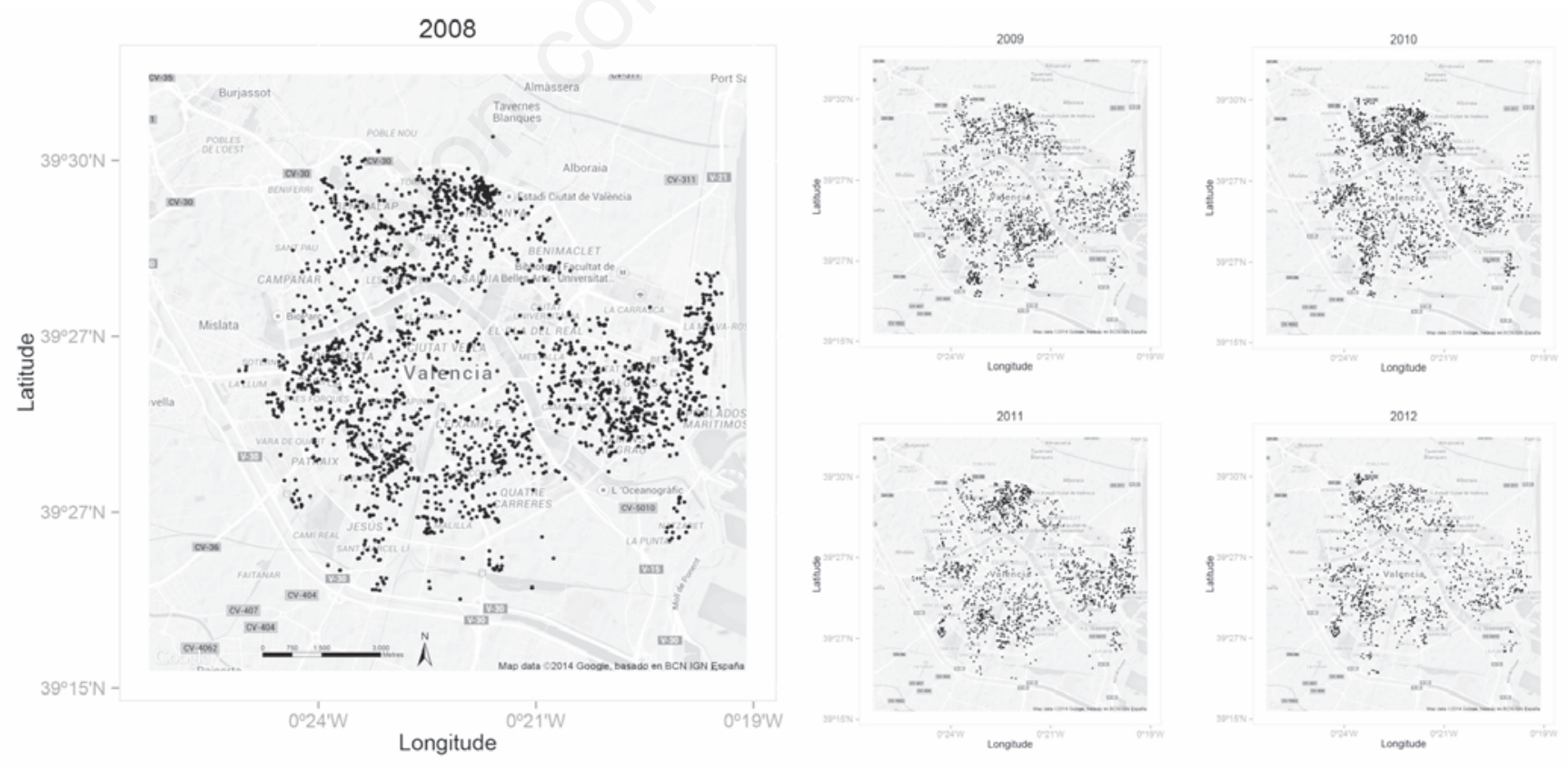

Figure 3. Spatial distribution of varicella cases for the time period 2008-2012. 


\section{Space-time analysis}

The values of the STIK function calculated for the data $K_{S T}(u, v)$, and simulations under two null hypotheses are compared. First hypothesis of no spatio-temporal clustering (Figure 6A) and a second one of no spatio-temporal interaction (Figure 6B). The grey shading in Figure 6 A demarcates those values of $(v, v)$ for which the estimation of $k_{s t}(v$, $v$ ) lies above the $95^{\text {th }}$ percentile of estimates calculated from 500 simulations of an inhomogeneous Poisson process. This indicates spatiotemporal clustering to a distance up to $500 \mathrm{~m}$ and a time-lag of 10 weeks, and it also suggests the existence of spatio-temporal clustering for distances from $500 \mathrm{~m}$ to $1 \mathrm{~km}$ and a time-lag between 3 and 10 weeks.

For the second null hypothesis, concerning the spatio-temporal interaction we compared the values of the STIK function with the tolerance envelopes calculated by randomly relabelling the locations holding the times fixed. The black shading in Figure 6B corresponds to the comparison statistic $K_{S T}(u, v)-K_{S}(u) K_{T}\left(v_{s}\right.$. This indicates spatio-temporal interaction at distances up to $500 \mathrm{~m}$ and time-lags up to 10 weeks and also at distances from $500 \mathrm{~m}$ to $1 \mathrm{~km}$ and time-lags up to 3 weeks.

These finding are consistent with the opinion of the epidemiologists that varicella cases are close in space, because of the highly contagious factor, and that temporal clustering could be found at time-lags higher than 4 weeks.

\section{Clustering localization}

Using the information provided by the previous method we ran a retrospective space-time analysis scanning for clusters with high rates using the StatScan method (Kulldorff, 2010). Weekly cases were analysed, from the first week of 2008 to the last week of 2012, giving a total of 260 weeks. We identified each case by its geographical location within the study area, the city of Valencia.

The previous analysis gave us the information that spatio-temporal clusters can be found at distances up to $1 \mathrm{~km}$ and time lags up to 10 weeks. Thus, we considered maximum spatial cluster size $25 \%$ of the population at risk, a circle with one kilometre radius, maximum temporal cluster size of 10 weeks and performed a standard Monte Carlo with 999 replications. There were a total of 19 significant clusters identified by the scan statistic having $\mathrm{P}<0.05$. On average, 3 clusters were detected each year. The average cluster radius is $0.57 \mathrm{~km}$, with a maximum of $0.98 \mathrm{~km}$ and a minimum of $0.054 \mathrm{~km}$. The smallest cluster was registered in the West of the city of Valencia beginning in March of 2009 (lasts for 8 weeks) and encompassing an area with radius of $0.054 \mathrm{~km}$. The largest cluster is 8 weeks long (begins in March of 2011), was located in the East of the city encompassing an area with radio of 0.98 $\mathrm{km}$ (extends outside the city). The average duration of the clusters was approximately 7 weeks, with a minimum of 2 weeks and a maximum of 9 weeks.

Figure 7 shows six most likely space-time clusters identified using the scan method. The most likely cluster (the main cluster) has a radius of $0.54 \mathrm{~km}$ and a time frame of 9 weeks, from week 148 to week 157, corresponding to the end of 2010 (November and December). There were 99 observed cases in this cluster area during the time frame window, compared to the expected 20.28 resulting a 4.88 ratio. Clustering across years showed consistent patterns, the spatial locations surrounding the center of the city. Both spatio-temporal clusters

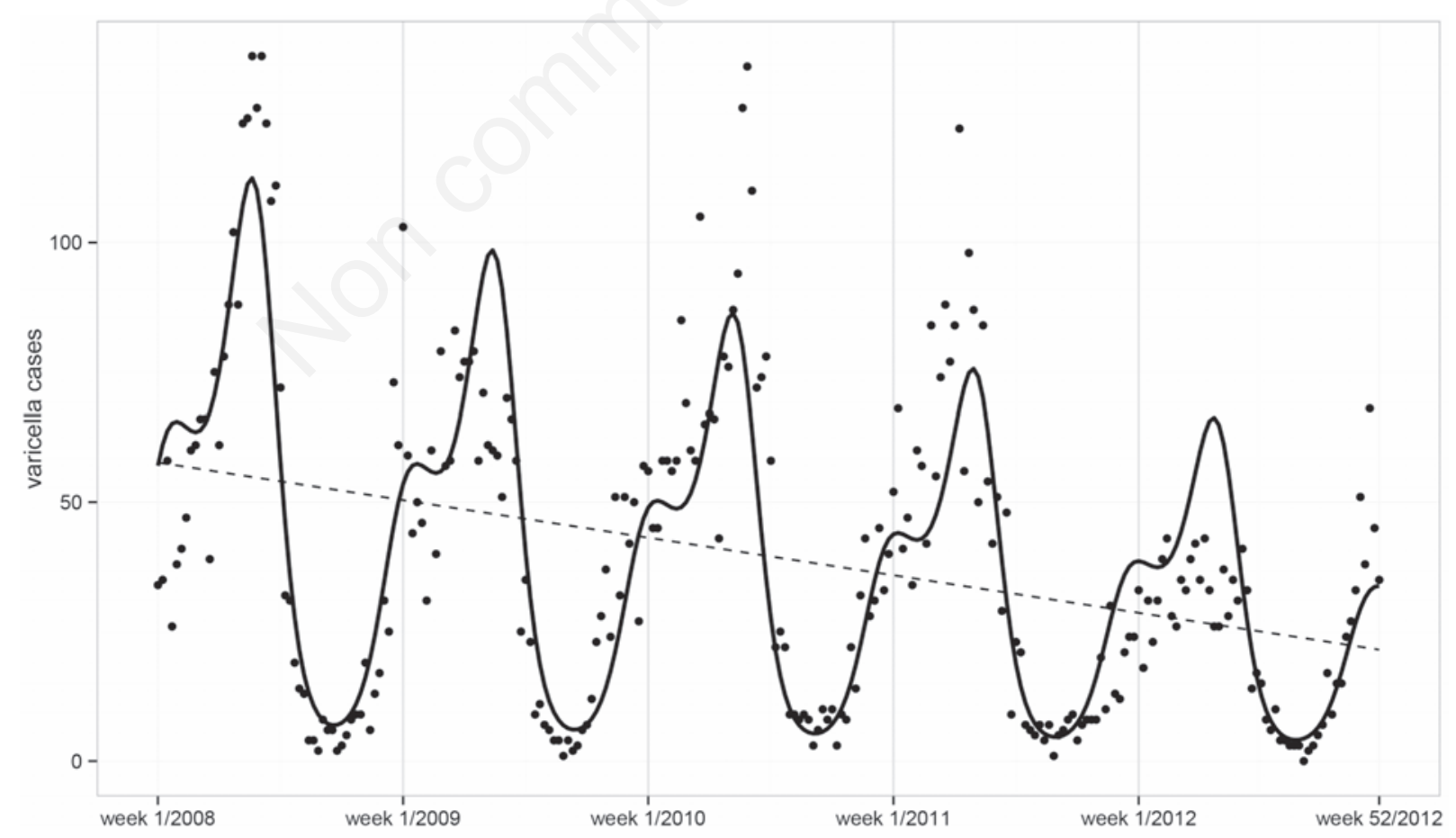

Figure 4. Weekly reports of varicella cases $(\bullet)$, fitted regression curve (-) and general lineal trend (----). 

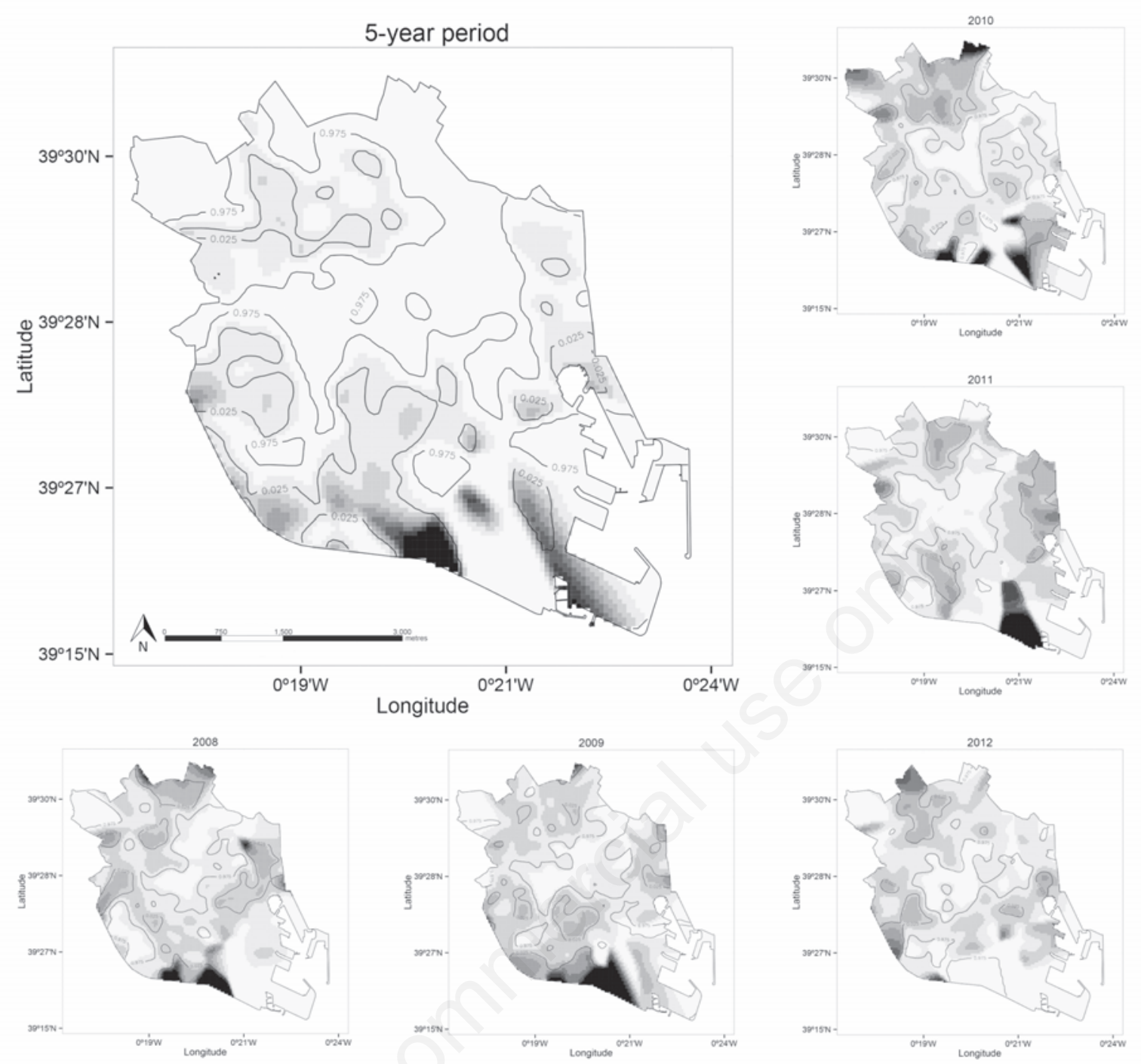

Figure 5. Local $P$ values of the Monte Carlo test for spatial clustering for 2008-2012 and for each year separately.
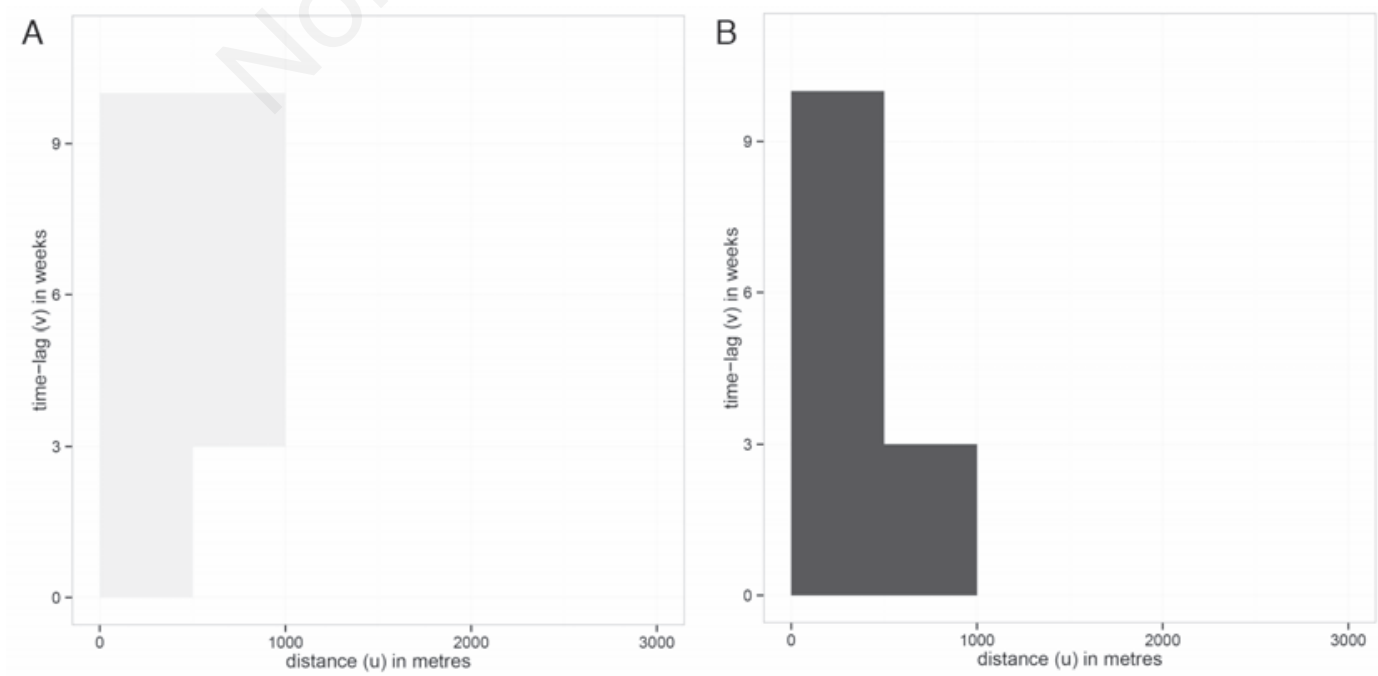

Figure 6. Comparison between test statistics and tolerance envelopes indicating spatio-temporal clustering [grey shading (A)] and spatio-temporal interaction [black shading $(B)]$. 
detected using the StatScan software and purely spacial clusters, are located on the peripheral area of the city.

\section{Discussion}

We examined temporal, spatial and spatio-temporal methods for the distribution of the incidence of varicella cases in Valencia, Spain. We found that varicella data has a decreasing temporal trend, a pronounced 12-month periodicity and two complementary periods. Several areas with a significant increment in the number of cases surrounding the center of the city were observed and space-time aggregation for distances of up to $1 \mathrm{~km}$ and a time-lag of up to 10 weeks was established. We observed space-time interaction and localized space-time clusters using the SatScan software.

According to the opinion held by epidemiologists, the core transmis- sion of the chickenpox virus could be represented by the schools. In Valencia there is often a correspondence between home and school, since when applying for both public and subsidised schools, the assignment is mainly by residence. Most private schools, including bilingual ones, are located on the outskirts of Valencia (except for the German school), so they are not included in the geographic area of our study.

From 2006-2008 to 2013, the Autonomous Communities of Madrid and Navarra and the cities of Ceuta and Melilla established universal childhood vaccination from 12 to 15 months of age. This pattern of vaccination was also recommended by the Spanish Association of Pediatrics, so many parents throughout the study period (2008-2012) purchased the vaccine on their own and had their children vaccinated at that age, especially in families with a medium or medium-high cultural and socioeconomic status. The areas where we identified spatiotemporal clusters correspond to areas in the city of Valencia where the population is economically disadvantaged or perhaps less educated and less aware of vaccination schedules.

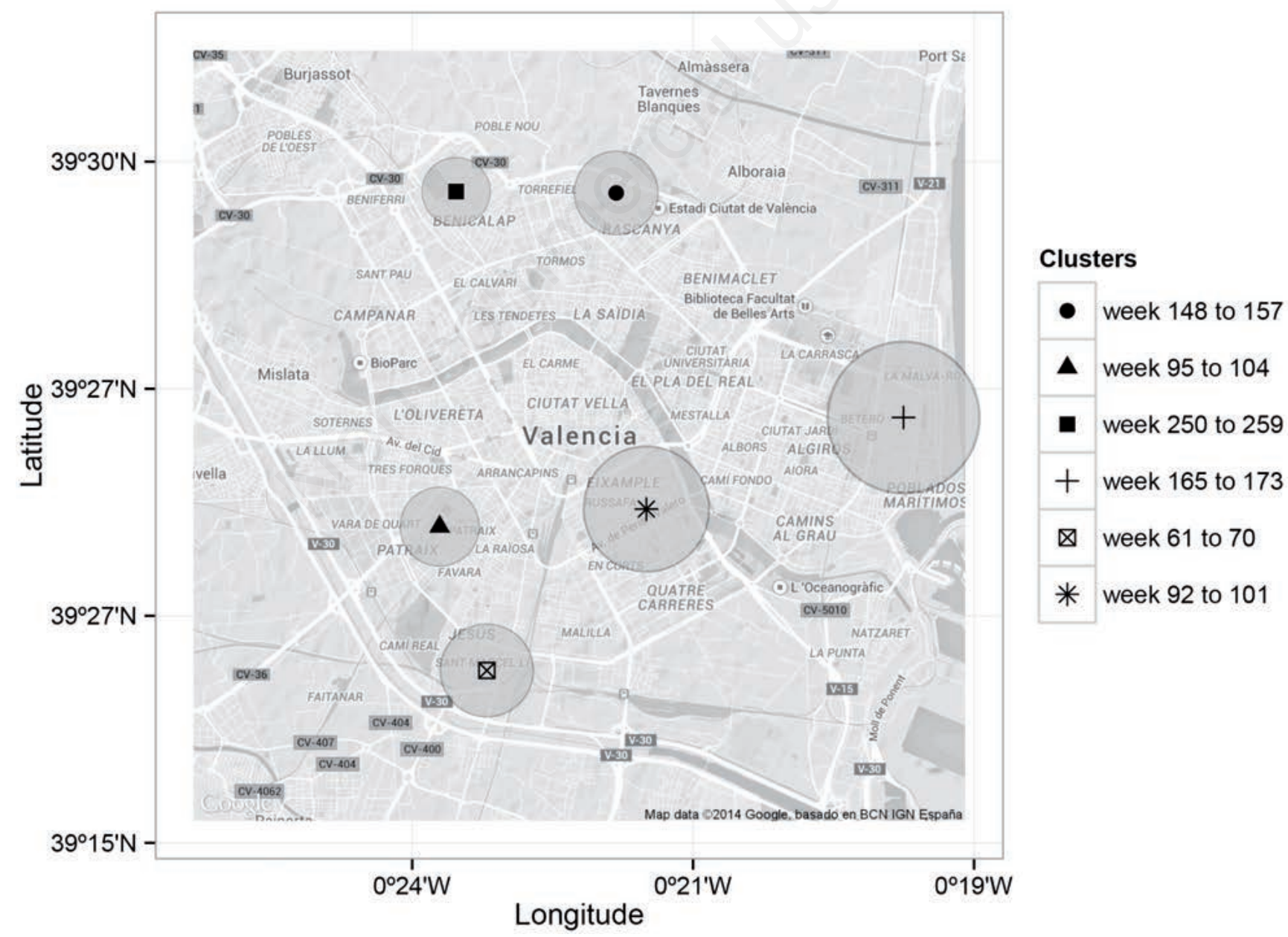

Figure 7. The six most likely spatio-temporal clusters identified using SatScan software. 


\section{Conclusions}

This paper represents a starting point for our future work and exemplifies an exploratory analysis of a space-time point pattern. Empirical and mechanistic models will be considered to continue this work.

\section{References}

Al-Tawfiq JA, Abukhamsin A, Memish ZA, 2013. Epidemiology and impact of varicella vaccination: a longitudinal study 1994-2011. Travel Med Infect Dis 11:310-4.

Baddeley A, Moller J, Waagepetersen R, 2010. Non-and semi-parametric estimation of interaction in inhomogeneous point patterns. Stat Neerl 54:329-50.

Brisson M, Edmunds W J, Law B, Gay N J, Walld R, Brownell M, Roos L, De Serres G, 2001. Epidemiology of varicella zoster virus infection in Canada and the United Kingdom. Epidemiol Infect 127:305-14.

Diggle PJ, 2014. Statistical analysis of spatial and spatio-temporal point patterns. CRC Press, Boca Raton, FL, USA.

Diggle PJ, Zheng P, Durr P, 2005. Nonparametric estimation of spatial segregation in a multivariate point process: bovine tuberculosis in Cornwall, UK. J R Stat Soc C App 54:645-8.
Gabriel E, Diggle P J, 2009. Second-order analysis of inhomogeneous spatio-temporal point process data. Stat Neerl 63:43-51.

Gabriel E, Wilson DJ, Leatherbarrow AJH, Cheesbrough J, Gee S, Bolton E, Fox A, Fearnhead P, Hart CA, Diggle PJ, 2010. Spatio-temporal epidemiology of Campylobacter jejuni enteritis, in an area of Northwest England, 2000-2002. Epidemiol Infect 138:1384-90.

Gil-Prieto R, Walter S, Gonzalez-Escalada A, Garcia-Garcia L, MarínGarcía P, Gil-de Miguel A, 2014. Different vaccination strategies in Spain and its impact on severe varicella and zoster. Vaccine 32:27783.

Kulldorff M, 2010. SatScan user guide for version 9.0. Availble from: www.satscan.org/

Ozaki T, 2013. Long-term clinical studies of varicella vaccine at a regional hospital in Japan and proposal for a varicella vaccination program. Vaccine 31:6155-60.

Peña Rey I, De Aragón MVM, Hueso AV, Arellano MT, Cabero EA, Rodríguez BS, 2009. Epidemiology of varicella in Spain pre-and post-vaccination periods. Rev Esp Salud Public 83:711-24.

Ripley B, 1981. Spatial statistics. Wiley, New York, NY, USA.

Servicio de Vigilancia y Control Epidemiológico, 2007. Protocolos ED0. Generalitat Valenciana. Conselleria de Sanitat. Dirección General de Salud Pública. Servicio de Vigilancia y Control Epidemiológico, Valencia, Spain

Statistics Office of Valencia Local Council, 2010. Municipal register of inhabitants at 1st of January. Valencia Local Council, Valencia, 